\title{
Colorectal Cancer Chemotherapy during COVID-19 Pandemic
}

\begin{abstract}
The management of patients with colorectal cancer during the current SARS-CoV2 pandemic opens a Pandora's Box. While the world is facing an unprecedented crisis of fighting a life-threatening infectious disease, patients with colorectal cancer are facing the dual challenge to fight cancer while protecting them from infection. We attempted to critically examine the existing evidence for chemotherapy in colorectal cancer in different stages of disease and suggest treatment options in these vulnerable patients. Treatment options which do not overburden existing health-care resources can be provided for patients with colorectal cancer patients requiring chemotherapy without significant compromise in efficacy or increase the risk of hospital acquired SAR-CoV-2 infection.
\end{abstract}

Keywords: Chemotherapy, colorectal cancer, COVID-19, SARS CoV2

\section{Introduction}

Colorectal cancer is one of the five most common cancers worldwide and in India. ${ }^{[1]}$ The onset of SARS-CoV-2 pandemic worldwide had led to a lockdown in many parts of the world, with more than 1.2 million people affected and 65,000 deaths reported at the time of writing this article. The pandemic is expected to grow further affecting most of the countries. The management of patients with colon cancer needs to be re-evaluated without overburdening the existing health-care resources. The benefit of anti-cancer treatment should be weighed against the risk of infection with SARS-CoV-2 due to hospital visits. Cancer patients are at a high risk of adverse outcomes (need for hospitalization, admission to intensive care units) and death from SARS-CoV-2. ${ }^{[2]}$ The presence of comorbidities such as cardiovascular diseases, hypertension, diabetes, chronic respiratory illness worsens the outcome of SARS-CoV-2 infection [Table 1]. ${ }^{[3]}$ Age is another risk factor and the risk of mortality based on age is presented in Table $2 .{ }^{[4]} \mathrm{We}$ attempt to re-evaluate existing evidence and formulate a treatment strategy in colorectal cancer to decrease risk of infection during this pandemic without compromising oncological outcomes.

This is an open access journal, and articles are distributed under the terms of the Creative Commons Attribution-NonCommercial-ShareAlike 4.0 License, which allows others to remix, tweak, and build upon the work non-commercially, as long as appropriate credit is given and the new creations are licensed under the identical terms.

For reprints contact: WKHLRPMedknow_reprints@wolterskluwer.com

\section{Adjuvant Chemotherapy in Colon Cancer}

The absolute benefit of adjuvant chemotherapy is $5 \%$ in resected colon cancer. ${ }^{[5]}$ However, the benefit varies based on the stage of the disease and clinicopathologic features. Patients with stage 1 are advised only observation. Benefits of chemotherapy based on stage and age are summarized in Table 3.

\section{High Risk Stage 2 Colon Cancer}

High risk features in a resected Stage 2 colon cancer include T3/T4 disease, ${ }^{[6]}<12$ lymph nodes resected in surgical specimen, poorly differentiated adenocarcinoma, lymphovascular invasion and perineural invasion, obstruction or perforation at presentation. However, all these high-risk features have differential impact on outcome. Patients with high microsatellite instability (MSI-H) do not benefit from single-agent fluoropyrimidine-based chemotherapy. ${ }^{[7]}$ However, MSI-H patients with T4 disease may require oxaliplatin-based chemotherapy

Submitted: 09-Apr-2020

Revised: 17-Apr-2020

Accepted: 19-Apr-2020

Published: 26-May-2020

Address for correspondence:

Dr. Sainath Bhethanabhotla, Department of Medical

Oncology, Care Hospitals, Hyderabad,

Telangana, India.

E-mail: bsainath101@yahoo.co.in

How to cite this article: Bhethanabhotla $S$, Pramanik R, Srivastava P, Mehta P, Patel A, Biswas B, et al. Colorectal cancer chemotherapy during COVID-19 pandemic. Indian J Med Paediatr Oncol 2020;41:156-60.

\section{Sainath}

Bhethanabhotla ${ }^{1}$, Raja Pramanik², Priyanka Srivastava ${ }^{3}$, Prashant Mehta ${ }^{4}$, Amol Patel ${ }^{5}$, Bivas Biswas ${ }^{6}$, Atul Batra², Vineet Govinda Gupta ${ }^{7}$, Chandan Das ${ }^{8}$, Shubh Mahindru ${ }^{9}$

${ }^{1}$ Department of Medical Oncology, Care Hospitals, Hyderabad, Telangana, India, ${ }^{2}$ Department of Medical Oncology, All India Institute of Medical Sciences, New Delhi, India, ${ }^{3}$ M.S. Patel Cancer Center, Shreekrishna Hospital and Medical Research Centre, Karamsad, Gujarat, India, ${ }^{4}$ Department of Medical Oncology, Asian Institute of Medical Sciences, Faridabad, Haryana, India, ${ }^{5}$ Malignant Diseases Treatment Centre, Army Hospital Research and Referral, New Delhi, India, ${ }^{6}$ Department of Medical Oncology, Tata Medical Center, Kolkata, West Bengal, India, ${ }^{7}$ Department of Medical Oncology, Artemis Hospital, Gurgaon, Haryana, India, ${ }^{8}$ Regional Cancer Centre, Postgraduate Institute of Medical Education and Research, Chandigarh, India, ${ }^{9}$ Department of Surgical Oncology, Ivy Hospital, Mohali, Punjab, India

Access this article online Website: www.ijmpo.org DOI: 10.4103/ijmpo.ijmpo_149_20 Quick Response Code:

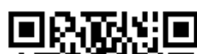

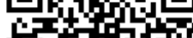
pris

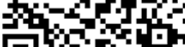
口+rats. 
regimen. ${ }^{[7]}$ Patients with microsatellite stable disease (MSS) and with T4 disease or more than one risk factor can be considered for oxaliplatin-based chemotherapy. We recommend oral capecitabine and oxaliplatin combination therapy (CAPOX) in this setting due to decreased hospital admission duration and decreased hospital visits as compared to infusional 5-fluorouracil (5-FU)-based regimen The recent IDEA meta-analysis in high risk Stage 2 colon cancer, which included 4 randomized controlled trials, showed 3-month duration of chemotherapy is as effective as 6 months especially when CAPOX (5 years disease-free survival [DFS] $82 \%$ vs. $81.7 \%$ for 6 months vs. 3 months) is used as the regimen. ${ }^{[8]}$ While the noninferiority did not reach statistical significance, the marginal decrease in absolute benefit does not outweigh the risk of adverse outcomes in these extraordinary situations. In patients with high risk Stage $2 \mathrm{MSS}$ disease we recommend 6 months of oral capecitabine or 3 months of CAPOX regimen, wherever applicable. In patients receiving combination chemotherapy, the maximum benefit is conferred with 5-FU/oral capecitabine as compared to oxaliplatin. In resource constrained setting, we would strongly recommend 6 months of oral capecitabine in these patients.

\section{Stage 3 Low Risk (T1-T3 and N1) Colon Cancer}

Recent IDEA meta-analysis and meta-analysis by Boyne et al. (which included randomized and nonrandomized trials) showed that patients with low risk Stage 3

\begin{tabular}{lcc}
\hline $\begin{array}{c}\text { Table 1: Risk of death/intensive care admission/ } \\
\text { mechanical ventilation due to comorbidities in severe } \\
\text { acute respiratory syndrome-coronavirus } 2\end{array}$ \\
\multicolumn{3}{c}{$\begin{array}{c}\text { HR for composite } \\
\text { end point }\end{array}$} \\
\hline \multicolumn{3}{c}{$\mathbf{9 5 \%}$ CI } \\
\hline Type of co morbidities \\
COPD \\
Type 2 diabetes mellitus & 2.681 & $1.424-5.048$ \\
Hypertension & 1.586 & $1.028-2.449$ \\
Active malignancy & 1.575 & $1.069-2.322$ \\
Age & 3.501 & $1.604-7.643$ \\
Smoking & 1.030 & $1.022-1.050$ \\
Number of comorbidities & 1.668 & $1.006-2.764$ \\
1 & 1.789 & $1.155-2.772$ \\
2 or more & 2.592 & $1.611-4.171$ \\
\hline CI - Confidence interval; COPD - Chronic obstructive pulmonary \\
disease; HR: Hazard ratio
\end{tabular}

(T1-T3, N1) had non inferior outcome with 3 months of adjuvant CAPOX chemotherapy. ${ }^{[9]}$ Patients already on oxaliplatin-based regimen can receive 3 months of adjuvant chemotherapy without compromising long-term outcome. ${ }^{[9,10]}$ However, among the two active agents, 5-FU/oral capecitabine adds substantial benefit to surgery as compared to oxaliplatin. Hence, in resource constrained situations, oral capecitabine is strongly recommended in these patients and resumption of oxaliplatin in subsequent cycles for a total of 4 cycles may be an optimum strategy in these circumstances despite lack of Level 1 evidence. ${ }^{[1]}$

\section{Stage 3 High-Risk (T4 or N2 Disease) Colon Cancer}

These patients require 6 months of adjuvant chemotherapy. It is preferable to use CAPOX regimen over FOLFOX due to the shorter infusion time, decreased frequency of visits ( 3 weeks vs. 2 weeks), and duration of hospital stay ( $4 \mathrm{~h}$ vs. $48 \mathrm{~h}$ ) with similar benefit. The time to recurrence in this subgroup of patients is 14 months according to ACCENT database, ${ }^{[12]}$ and hence, we strongly recommend continuing chemotherapy in these patients for a total duration of 6 months. An informed decision needs to be taken after discussing the risks and benefits of chemotherapy in the background of the pandemic with the patient and their caregiver (s). In high risk (T4/N2), most of the benefit is already achieved with 4 cycles of CAPOX so in extenuating circumstances, an individualized risk-benefit decision may be taken for continuing 4 more cycles. The hazard ratio for recurrence in T4/N2 is only 1.12 and the number needed to treat to reduce one extra recurrence is 59. ${ }^{[13]}$ In case of significant health system strain and risk of COVID-19 infection, the patient can switch to oral capecitabine alone after 4 cycles CAPOX.

\section{Adjuvant Chemotherapy in Rectal Cancer}

Adjuvant chemotherapy in rectal cancer has always been debated in terms of benefit. However, the principles of adjuvant chemotherapy may not vary between colon cancer and rectal cancer.

Patients with a good response (defined as pathological complete response and down staging to $\leq$ ypT2 and node negative disease) are likely to benefit from adjuvant chemotherapy. This is based on the large data from a retrospective study which showed patients with baseline

\begin{tabular}{lccc}
\hline \multicolumn{4}{c}{ Table 2: Age-wise mortality rates with COVID-19 } \\
\hline Age (years old) & Death rate confirmed cases (\%) & Death rate in China (\%) & Death rate in Italy (\%) \\
\hline $80+$ & 21.9 & 14.8 & 20.2 \\
$70-79$ & & 8.0 & 12.8 \\
$60-69$ & & 3.6 & 3.5 \\
$50-59$ & & 1.3 & 1.0 \\
$40-49$ & 0.4 & 0.4 \\
$30-39$ & & 0.2 & 0.3 \\
\hline
\end{tabular}




\begin{tabular}{|c|c|c|c|}
\hline \multicolumn{4}{|c|}{$\begin{array}{l}\text { Table 3: Benefit of adjuvant chemotherapy in resected } \\
\text { colon cancer based on stage and age }\end{array}$} \\
\hline \multicolumn{4}{|c|}{$\begin{array}{l}\text { Average benefit of adjuvant 5-fluorouracil (high-grade Stage 2) } \\
\text { percentage of patients alive due to chemotherapy after } 5 \text { years }\end{array}$} \\
\hline \multirow[t]{2}{*}{ Stage } & \multicolumn{3}{|c|}{ Age } \\
\hline & $30-40(\%)$ & $40-60(\%)$ & $60-80(\%)$ \\
\hline T3N0 & 2.2 & 2.2 & 1.9 \\
\hline \multicolumn{4}{|c|}{$\begin{array}{l}\text { Average benefit of adjuvant FOLFOX percentage of patients } \\
\text { alive due to chemotherapy after } 5 \text { years (T4 disease and stage } 3 \text { ) }\end{array}$} \\
\hline T4N0 & 3.9 & 3.9 & 3.4 \\
\hline T3N1 & 16.9 & 16.6 & 14.4 \\
\hline T3N2 & 24.5 & 24.1 & 20.9 \\
\hline T3N3 & 26.7 & 26.3 & 22.9 \\
\hline T4N1 & 21.9 & 21.5 & 18.7 \\
\hline T4N2 & 27.5 & 27.1 & 23.6 \\
\hline T4N3 & 18.9 & 18.6 & 16.1 \\
\hline
\end{tabular}

Data compiled using onco-assist application endorsed by ESMO

clinical T3/4 disease and lymph node positive disease who achieved pathological complete response benefitted most from adjuvant chemotherapy. ${ }^{[14]}$ Meta-analysis by Maas et al. showed significant benefit from adjuvant chemotherapy in patients achieving ypT1-T2 postneoadjuvant chemoradiation. ${ }^{[15]}$ Most of the studies in this meta-analysis used single-agent fluoropyrimidine as adjuvant chemotherapy. Since oral capecitabine monotherapy obviates the need for hospital admission, we would suggest oral regimen in these patients without affecting long-term survival.

Patients with no or minimal response to neoadjuvant chemoradiation (ypT3/T4 and node positive) would benefit from oxaliplatin-based chemotherapy. Meta-analysis by Zhao et al. showed the maximum benefit from combination chemotherapy in this setting. ${ }^{[16]}$ However, there was no benefit in overall survival with further need for longer follow-up. In view of high risk of death due to disease in these nonresponders $(68 \%$ vs. $57 \%$ in patients receiving adjuvant chemotherapy), there is a strong reason to use oxaliplatin-based combination chemotherapy. ${ }^{[17]}$ Similar to patients with high risk Stage 3 colon cancer, these patients should be treated with CAPOX-based chemotherapy which decreases the need for hospital visit and duration of hospital stay.

\section{Neoadjuvant Chemotherapy in Colon Cancer Patients Awaiting Surgery}

Patient with potentially resectable disease but deferred surgery due to resource constraints which includes transport, availability of hospital beds and personal protective equipment supply, and risk of infection in the perioperative period can be started on neoadjuvant chemotherapy. The recent FOxTROT study examined the role of neoadjuvant chemotherapy in resectable colon cancer which showed histological regression in $59 \%$ of patients. ${ }^{[18]}$ There was decreased rate of incomplete resection in patients with
cT3/T4 disease without the risk of disease progression in this study, though there was no improvement in DFS. Oxaliplatin-based regimen preferably CAPOX can be used in patients awaiting curative surgery.

\section{Metastatic Colorectal Malignancy}

Newly diagnosed patients with metastatic disease can undergo chemotherapy with CAPOX regimen along with biological agents. Irinotecan-based regimens, which use infusional 5-FU, require prolonged hospitalization and frequent visits, can be avoided. If infusional regimen is used, portable pump can be used to decrease the duration of hospitalization.

Biological agents (cetuximab, panitumumab, and bevacizumab) add incremental survival benefit when added to chemotherapy. Anti-epidermal growth factor receptor agents cetuximab or panitumumab have demonstrated survival benefit with only infusional 5-FU regimen in left-sided RAS wild type colon cancer. An informed decision should be taken after discussing the risks and benefits of infusional regimen and need for frequent hospital visits with patient and their caregiver (s). Portable pumps which reduce the duration of hospitalization can be used when infusional regimen is given. Patients with right-sided tumor and left-sided tumor with RAS mutation bevacizumab is the preferred biological agent which can be used with CAPOX regimen.

Patients with metastatic disease and who previously received oxaliplatin-based regimen can be considered for modified capcitabine and irinotecan or tegafur, leucovorin and irinotecan (1:4 molar combination of ftorafur with uracil, leucovorin and irinotecan) rather than infusional 5 $\mathrm{FU}$ and irinotecan-based regimen (FOLFIRI) ${ }^{[19]}$ A phase 2 study by Shigeta et al. showed similar progression free survival with both TEGAFIRI and FOLFIRI regimen (9.9 months vs. 10.6 months). ${ }^{[20]}$ The use of third or more line (e.g., checkpoint inhibitors, anti-Her2 therapy, anti-BRAF) should be done extremely judiciously on case by case basis explaining the risks and chances of benefit. Palliative surgery for perforation and obstruction may be done while biopsies of metastatic lesions should be minimized and liquid biopsy used.

Patients with poor performance status and not fit for intensive chemotherapy should be encouraged for home-based care or single agent oral fluoropyrimidine (capecitabine, UFT), if feasible. In general, the bolus part of modified FOLFOX 6 may be omitted and the infusional 5FU continued, in an attempt to minimize neutropenia. In the metastatic setting, cycles may be delayed judiciously to decrease the frequency of visits. Granulocyte colony stimulating factors may be used liberally in patients with previous or expected toxicity.

UFT-based combination regimen with either oxaliplatin or irinotecan along with or without biological agent has 


\begin{tabular}{|c|c|c|}
\hline & Treatment options & Suggested alternatives \\
\hline \multicolumn{3}{|l|}{ Adjuvant chemotherapy in colon cancer } \\
\hline \multicolumn{3}{|l|}{ High risk stage 2} \\
\hline MSI-H pT4 only & CAPOX 3 months & Observation or 3 months CAPOX \\
\hline MSS & SA Capecitabine/CAPOX/FOLFOX & $\begin{array}{l}\text { Oral Capecitabine for } 6 \text { months (preferred) } / \text { no }>3 \\
\text { months CAPOX }\end{array}$ \\
\hline \multirow[t]{2}{*}{ Low risk stage 3} & CAPOX 3 months & CAPOX 3 months \\
\hline & FOLFOX 6 months & Oral capecitabine (6 months) \\
\hline \multirow[t]{2}{*}{ High risk stage 3} & CAPOX 6months & CAPOX 6 months \\
\hline & FOLFOX 6 months & Oral Capecitabine 2 cycles f/b CAPOX 6 cycles \\
\hline Elderly patients & SA capecitabine & Oral capecitabine \\
\hline \multicolumn{3}{|l|}{ Adjuvant chemotherapy in rectal cancer } \\
\hline \multirow[t]{3}{*}{ Good responders (pCR, ypT1-2) } & CAPOX & Oral capecitabine \\
\hline & FOLFOX & \\
\hline & SA Capecitabine & \\
\hline \multirow[t]{2}{*}{ Poor responders } & CAPOX & CAPOX 3months \\
\hline & FOLFOX & Oral Capecitabine 6 months \\
\hline \multicolumn{3}{|l|}{ Metastatic colorectal cancer } \\
\hline \multirow[t]{4}{*}{ Treatment naïve } & CAPOX & CAPOX \\
\hline & FOLFOX & Modified XELIRI \\
\hline & FOLFIRI & TEGAFOX \\
\hline & & TEGAFIRI \\
\hline \multirow{2}{*}{$\begin{array}{l}\text { Postoxaliplatin-based chemotherapy } \\
\text { regimen }\end{array}$} & FOLFIRI & Modified XELIRI \\
\hline & & TEGAFIRI \\
\hline
\end{tabular}

shown similar efficacy to infusional regimen. ${ }^{[21-24]}$ The data for UFT-based combination chemotherapy in this setting are from Phase 2 studies; however, it is safer alternative when the risk of SARS-Cov2 infection and complications is high in the current situation.

Patients who attained partial response after certain cycles of chemotherapy can be advised maintenance therapy with oral capecitabine. ${ }^{[25]}$ Biological agents may be withheld for few cycles after explaining the risk and benefits of such approach during pandemic to patient and their caregiver (s). Table 4 summarizes the treatment suggestions based on the existing evidence depending on stage and clinical scenario. ESMO guidelines for the management of CRC in COVID19 pandemic are a useful resource in this setting and we agree with most of the recommendations. All the "high-priority" interventions should be given precedence over the "medium and low priority" ones. ${ }^{[25]}$ All treatment including first line for patients with poor performance status, heavy comorbidities, slow growing recurrent disease and those patients who had severe complications during the adjuvant therapy should be delayed.

Apart from these specific recommendations, general principles such as social-distancing in the outpatient departments, cough etiquettes, increased use of telemedicine to decrease hospital visits should be followed and explained to colorectal cancer patients.

The use of chemotherapy in the management of colorectal cancer during SARS-CoV2 pandemic requires prudent use of health-care resources and existing evidence. With prospects of clinical trials in these situations being difficult to conduct to give definitive evidence we attempted to derive the rational protocols from the existing data to formulate optimum treatment strategy without significantly deviating from the evidence.

Financial Support and Sponsorship

Nil.

\section{Conflicts of interest}

There are no conflicts of interest.

\section{References}

1. Global Cancer Statistics 2018: GLOBOCAN Estimates of Incidence and Mortality Worldwide for 36 Cancers in 185 Countries. Available from: https://acsjournals.onlinelibrary.wiley. com/doi/epdf/10.3322/caac. 21492. [Last accessed on 2020 Apr 05].

2. Liang W, Guan W, Chen R, Wang W, Li J, Xu K, et al. Cancer patients in SARS-CoV-2 infection: A nationwide analysis in China. Lancet Oncol 2020;21:335-7.

3. Guan W, Liang W, Zhao Y, Liang H, Chen Z, Li Y, et al. Comorbidity and its impact on 1590 patients with Covid-19 in China: A nationwide analysis. Eur Respir J 2020. pii: 2000547.

4. Onder G, Rezza G, Brusaferro S. Case-fatality rate and characteristics of patients dying in relation to COVID-19 in Italy. JAMA 2020. Published online March 23, 2020. Available from: https://jamanetwork.com/journals/jama/fullarticle/2763667. [Last accessed on 2020 Apr 09].

5. André T, Boni C, Navarro M, Tabernero J, Hickish T, Topham C, 
et al. Improved overall survival with oxaliplatin, fluorouracil, and leucovorin as adjuvant treatment in stage II or III colon cancer in the MOSAIC trial. J Clin Oncol 2009;27:3109-16.

6. Weiser MR. AJCC $8^{\text {th }}$ edition: Colorectal cancer. Ann Surg Oncol 2018;25:1454-5.

7. Sargent DJ, Marsoni S, Monges G, Thibodeau SN, Labianca R, Hamilton SR. Defective mismatch repair as a predictive marker for lack of efficacy of fluorouracil-based adjuvant therapy in colon cancer. J Clin Oncol 2010;28:3219-26.

8. Prospective pooled analysis of four randomized trials investigating duration of adjuvant (adj) oxaliplatin-based therapy (3 vs. 6 months $\{\mathrm{m}\}$ ) for patients (pts) with high-risk stage II colorectal cancer (CC). J Clin Oncol 2019;37 (15 suppl):3501. Available from: $\quad$ https://ascopubs.org/doi/abs/10.1200/JCO.2019.37.15_ suppl. 3501. [Last accessed on 2020 Apr 05].

9. Boyne DJ, Cuthbert CA, O’Sullivan DE, Sajobi TT, Hilsden RJ, Friedenreich CM, et al. Association between adjuvant chemotherapy duration and survival among patients with stage II and III colon cancer. JAMA Netw Open 2019;2:e194154.

10. Grothey A, Sobrero AF, Shields AF, Yoshino T, Paul J, Taieb J, et al. Duration of adjuvant chemotherapy for stage III colon cancer. N Engl J Med 2018;378:1177-88.

11. Peng J, Li W, Zhang R, Lin J, Tang J, Wen Y, et al. Safety and efficacy of a modified XELOX adjuvant regimen for patients with operated stage III colon cancer: A Chinese single-center experience. Cancer Commun (Lond) 2019;39:59.

12. Shah MA, Renfro LA, Allegra CJ, André T, de Gramont A, Schmoll HJ, et al. Impact of patient factors on recurrence risk and time dependency of oxaliplatin benefit in patients with colon cancer: Analysis from modern-era adjuvant studies in the adjuvant colon cancer end points (ACCENT) database. J Clin Oncol 2016;34:843-53.

13. Lieu C, Kennedy EB, Bergsland E, Berlin J, George TJ, Gill S, et al. Duration of oxaliplatin-containing adjuvant therapy for stage III colon cancer: ASCO clinical practice guideline. J Clin Oncol 2019;37:1436-47.

14. Polanco PM, Mokdad AA, Zhu H, Choti MA, Huerta S. Association of adjuvant chemotherapy with overall survival in patients with rectal cancer and pathologic complete response following neoadjuvant chemotherapy and resection. JAMA Oncol 2018;4:938-43.

15. Maas $\mathrm{M}$, Nelemans PJ, Valentini V, Crane $\mathrm{CH}$, Capirci $\mathrm{C}$, Rödel C, et al. Adjuvant chemotherapy in rectal cancer: Defining subgroups who may benefit after neoadjuvant chemoradiation and resection: A pooled analysis of 3,313 patients. Int $\mathrm{J}$ Cancer 2015; 137:212-20.

16. Oxaliplatin/Fluorouracil-Based Adjuvant Chemotherapy for Locally Advanced Rectal Cancer after Neoadjuvant
Chemoradiotherapy and Surgery: A Systematic Review and Meta-Analysis of Randomized Controlled Trials-Zhao-2016-Colorectal Disease-Wiley Online Library. Available from: https://onlinelibrary.wiley.com/doi/epdf/10.1111/ codi. 13381. [Last accessed on 2020 Apr 05].

17. Valentini V, van Stiphout RG, Lammering G, Gambacorta MA, Barba MC, Bebenek M, et al. Nomograms for predicting local recurrence, distant metastases, and overall survival for patients with locally advanced rectal cancer on the basis of European randomized clinical trials. J Clin Oncol 2011;29:3163-72.

18. FOxTROT: An international randomised controlled trial in 1052 patients (pts) evaluating neoadjuvant chemotherapy (NAC) for colon cancer. J Clin Onco 2019;37 (15 Suppl):3504. Available from: https://ascopubs.org/doi/abs/10.1200/JCO.2019.37.15 suppl. 3504. [Last accessed on 2020 Apr 05].

19. $\mathrm{Xu} \mathrm{RH}$, Muro K, Morita S, Iwasa S, Han SW, Wang W, et al. Modified XELIRI (capecitabine plus irinotecan) versus FOLFIRI (leucovorin, fluorouracil, and irinotecan), both either with or without bevacizumab, as second-line therapy for metastatic colorectal cancer (AXEPT): A multicentre, open-label, randomised, non-inferiority, phase 3 trial. Lancet Oncol 2018;19:660-71.

20. Shigeta K, Hasegawa H, Okabayashi K, Tsuruta M, Ishii Y, Endo T, et al. Randomized phase II trial of TEGAFIRI (tegafur/uracil, oral leucovorin, irinotecan) compared with FOLFIRI (folinic acid, 5-fluorouracil, irinotecan) in patients with unresectable/recurrent colorectal cancer. Int J Cancer 2016;139:946-54.

21. Bennouna J, Saunders M, Douillard JY. The role of UFT in metastatic colorectal cancer. Oncology 2009;76:301-10.

22. Bajetta E, Di Bartolomeo M, Buzzoni R, Mariani L, Zilembo N, Ferrario E, et al. Uracil/ftorafur/leucovorin combined with irinotecan (TEGAFIRI) or oxaliplatin (tegafox) as first-line treatment for metastatic colorectal cancer patients: Results of randomised phase II study. Br J Cancer 2007;96:439-44.

23. Douillard JY, Zemelka T, Fountzilas G, Barone C, Schlichting M, Heighway $\mathrm{J}$, et al. FOLFOX4 with cetuximab vs. UFOX with cetuximab as first-line therapy in metastatic colorectal cancer: The randomized phase II FUTURE study. Clin Colorectal Cancer 2014;13:14-26.e1.

24. Sheikh HY, Valle JW, Waddell T, Palmer K, Wilson G, Sjursen A. Alternating irinotecan with oxaliplatin combined with UFT plus leucovorin (SCOUT) in metastatic colorectal cancer. Br J Cancer 2008;99:577-83.

25. Sonbol MB, Mountjoy LJ, Firwana B, Liu AJ, Almader-Douglas D, Mody K, et al. The role of maintenance strategies in metastatic colorectal cancer: A systematic review and network meta-analysis of randomized clinical trials. JAMA Oncol 2019;6:e194489. 